\title{
'Smart' biomaterials and osteoinductivity
}

\section{Barbara D. Boyan and Zvi Schwartz}

We thank Dr. Yuan and colleagues for their correspondence on our News \& Views article (Are calcium phosphate ceramics 'smart' biomaterials? Nat. Rev. Rheumatol. $7,8-9 ; 2011),{ }^{1}$ in which they clarify the composition of the calcium phosphate ceramic implants that they tested in sheep and dogs for the ability to support ectopic bone formation ('Smart' biomaterials and osteoinductivity. Nat. Rev. Rheumatol. doi:10.1038/nrrheum.2010.210-c1). ${ }^{2}$ The fact that in their original study ${ }^{3}$ they did indeed use materials without any addition of cells, pretreatment with any special media, or addition of bioactive proteins or peptides strengthens their interpretation that the formatting of the ceramics itself resulted in osteoinduction. This is an important observation because it indicates that biomaterials can be designed to achieve specific outcomes in vivo without the addition of biological components such as cell attachment peptides, tethered growth factors, or exogenous cells.

A number of factors may have contributed to the ability of the calcium phosphate particles tested in the Yuan et al. study ${ }^{3}$ to induce ectopic bone formation, including the chemical composition of the implants, their surface texture and chemistry, their three dimensional shape, and the spatial distribution of the particles in the tissue. It will be interesting going forward to learn what features of a biomaterial are critical for achieving specific biological outcomes in clinical applications.

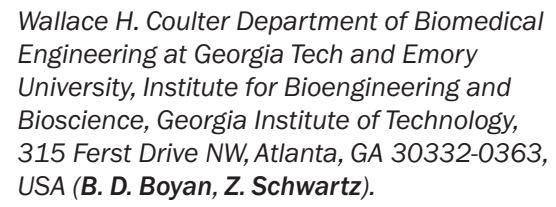

Correspondence to: B. D. Boyan barbara.boyan@bme.gatech.edu

\section{doi:10.1038/nrrheum.2010.210-c2}

\section{Competing interests}

B. D. Boyan and Z. Schwartz have received funding from the Musculoskeletal Transplant Foundation to study carriers for demineralized bone matrix, a grant from the NIH to support research on microstructured biomaterials and differentiation of osteoblasts and mesenchymal stem cells, and a grant from the US Department of Defense to develop technologies for stem cell delivery. B. D. Boyan has an issued US Patent for a method of making hydrogel implants (US Patent $7,682,540$ ), and B. D. Boyan and Z. Schwartz have filed a provisional patent with the US Patent and Trademark Office for novel methods of applying nanotexture to metal implants. B. D. Boyan has served as a consultant for ApaTech, Exactech, and Spineology, in the development of synthetic bone graft substitutes.

1. Boyan, B. D. \& Schwartz, Z. Are calcium phosphate ceramics 'smart' biomaterials? Nat. Rev. Rheumatol. 7, 8-9 (2011).

2. Yuan, H. et al. 'Smart' biomaterials and osteoinductivity. Nat. Rev. Rheum. doi:10.1038/nrrheum.2010.210-c1.

3. Yuan H. et al. Osteoinductive ceramics as a synthetic alternative to autologous bone grafting. Proc. Nat/ Acad. Sci. USA 107, 13614-13619 (2010). 